\title{
COMPARISON OF THE INTERNAL FIT OF CONVENTIONAL CASTING VERSUS CAD WAX (IN-VITRO STUDY)
}

\author{
Fatema Khaled Mansour*
}

\begin{abstract}
Statement of Problem: The accuracy of internal fit for subtractive CAD-CAM systems used in the fabrication of metal coping is still unclear.

Purpose: This in vitro study compared the internal fit of casting metal core using conventional wax versus CAD wax.

Material and Methods: Twenty four resin specimen dies were randomly divided into two groups according to method of metal coping construction using conventional (C) or CAD (D) wax. The silicon replica technique evaluated the internal fit using stereomicroscope at $\times 24$ magnification; the thickness of the silicon layer was measured at 20 reference points on each specimen. Parametric data of internal gap width was analyzed using one-way ANOVA.
\end{abstract}

Results: Different techniques of wax construction showed insignificant difference in internal gap values between specimens made with conventional (C) (103.89 \pm 40.39$)$ and CAD (D) waxing $(104.10 \pm 39.96)$. However, there was a significant difference between internal gap values measured at different sites.

Conclusions: Both construction techniques of casting were considered reliable in production within a clinically acceptable range regarding internal fit.

KEYWORDS: Internal fit, Lost wax technique, CAD wax, Conventional casting, Silicon replica.

Clinical Implications: Different wax fabrication techniques show similar internal values for casted core.

\section{INTRODUCTION}

The fit of a restoration is determined by the marginal and internal fit. Marginal gaps result in gingival irritation, cement dissolution and recurrent decay ${ }^{(3)}$. Internal fit is defined as the perpendicular distance between the framework and the abutment teeth and it is measured from occlusal/incisal and axial surfaces to the coping ${ }^{(1)}$. A range between 50 to $100 \mu \mathrm{m}$ has been considered acceptable ${ }^{(11)}$. Direct microscopic examination was commonly used followed by silicon replica technique that was

* Assistant Lecturer, Fixed Prosthodontics Department, Modern Science and Arts University, Faculty of Dentistry, Cairo, Egypt. 
observed under microscope as well. In addition, cross sectioning of cemented specimens could be done however; it is destructive ${ }^{(16)}$.

No enough scientific evidence has been reached on the accuracy of internal fit for different techniques for fabrication of metal coping using conventional and CAD wax ${ }^{(22)}$. This could reduce production cost, labor, and time therefore decreasing the errors and enhancing the accuracy.

The conventional technique for fabricating the metal substructure is the lost-wax technique. Conventional fabrication process involves multiple materials as well as clinical and laboratory stages, leading to unavoidable inaccuracies ${ }^{(1,2)}$. Wax up quality is dependent on the skill of the operator. Introduction of CAD/CAM systems, made it possible to fabricate the wax patterns from CAD blocks and omit several limitations. Using CAD/ CAM systems have many advantages such as producing higher and more uniform-quality restorations, standardizing restoration shaping and enhancing accuracy. However, CAD/ CAM systems also have some disadvantages, such as scanning systems finite resolution limitation which results in edges that are slightly rounded ${ }^{(1,3)}$.

The purpose of this study was to evaluate internal fit of conventionally constructed versus CAD wax. The null hypothesis was that there will be no difference in the internal fit and the measuring site of the internal fit along die/replica interface between different techniques of construction.

\section{MATERIALS AND METHODS}

A master stainless steel metal die was milled to resemble the preparation of mandibular first molar for construction of metal coping ${ }^{(13)}$. Metal die was duplicated to twenty four resin dies using polyvinylsiloxane duplication material (4) and randomly divided into two equal groups according to method of metal coping construction either using conventional (group C) or CAD (group D) wax.
According to previous studies ${ }^{(7,9,10)}$, twelve specimens in each group representing an $80 \%$ power to detect a difference between means of $40.81 \mu \mathrm{m}$. Sample size was calculated using power analysis software $\left(\mathrm{G}^{*} \text { Power }\right)^{(14)}$. After construction of resin dies by duplication from master metal die, they were randomly allocated equally to the two groups utilizing electronic website (www.random. org). After randomization, dies were assigned in opaque sealed envelopes by the supervisor. Blinding wasn't feasible for the operator because different techniques of restoration construction were used. Same assessor carried out all measurements and was blinded during measurements of internal fit to avoid performance bias. Also, statistician was blinded from knowing intervention and control to avoid detection bias during outcome assessment. It was assumed that new materials and techniques of construction could be faster and more accurate in producing restorations with better internal fit.

For the construction of casting, two types of wax were used in this study. Using the conventional wax, four coats of die spacer (each coat was $13 \mu \mathrm{m} \mathrm{X} 4$ $=52 \mu \mathrm{m}$ ) were applied on epoxy die and separating medium was applied to be able to remove the wax pattern. This was followed by dipping of die in wax heater dipping pot. Then sculpting grey opaque wax (GEO Crowax) was added to any thin areas by electric wax knife and margination with cervical red wax (GEO Crowax) to have precise margins. Verification with wax caliper to have $0.4 \mathrm{~mm}$ coping of wax thickness. While using CAD wax (Yamahachi), the epoxy die was fixed on scanning plate and scanned by Identica dental scanner after spraying with antireflection spray which has 3 axis of movement for accurate scanning. Then, images were transferred to create a three dimensional virtual die. Computer aided design (CAD) software Exocad program was used to digitally design the dental coping to have a thin uniform coping layer of $0.4 \mathrm{~mm}$ in thickness with 52 micron die spacer 
away $1 \mathrm{~mm}$ from margin equivalent to conventional wax used for standardization then milling CAD wax was done by five axis vhf K5 dry milling machine. For casting of metal substructure, $\mathrm{NiCr}$ alloy (Dentaurm) was used with phosphate bonded investment material.

For measuring the internal fit silicon replica technique $e^{(1,2,17)}$ was used using light body impression material. Putty impression material was used in order to hold the replica from the resin die surface. Each section had five reference points to be measured at different positions using stereo microscope at X24 magnification Figure (1). An overall of twenty reference points were measured in $\mu \mathrm{m}$ for each replica sample.

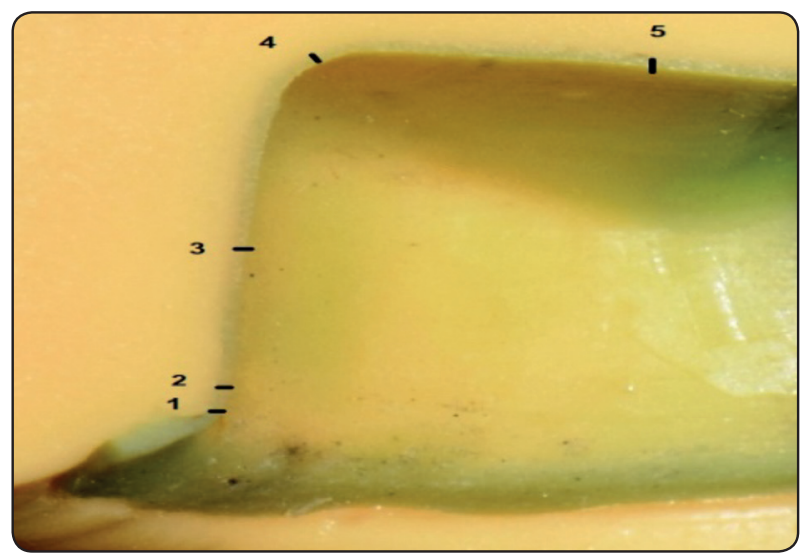

Fig. (1): Silicon replica specimen with five reference points for surface quadrant

\section{RESULTS}

Results were explored for normality by checking the data distribution, calculating the mean using Kolmogorov-Smirnov and Shapiro-Wilk tests. Parametric data of internal gap width were analyzed using one-way ANOVA followed by comparison of main and simple effects utilizing benferroni correction. The significance level was set at $\mathrm{P} \leq 0.05$ within all tests. Statistical analysis was performed with IBM® SPSS ${ }^{\circledR}$ Statistics Version 26 for Windows.
Numerical data were represented by mean and standard deviation (SD) values. The highest mean internal gap was apparent with CAD wax, while the lowest mean internal gap was found with conventional wax table (1).

\begin{tabular}{|c|c|c|c|}
\hline $\begin{array}{l}\text { Waxing } \\
\text { technique }\end{array}$ & Position & Mean \pm SD & $\mathrm{p}$-value \\
\hline \multirow{5}{*}{$\begin{array}{l}\text { 플 } \\
.0 \\
.00 \\
0 \\
0 \\
0 \\
0\end{array}$} & FL & $53.63 \pm 1.26$ & \multirow{5}{*}{$<0.001 *$} \\
\hline & Above FL & $71.04 \pm 2.12$ & \\
\hline & Axial & $101.01 \pm 6.09$ & \\
\hline & Axio-occlousal & $128.9 \pm 4.52$ & \\
\hline & Occlusal & $164.27 \pm 2.86$ & \\
\hline \multirow{5}{*}{ 远 } & FL & $53.69 \pm 1.36$ & \multirow{5}{*}{$<0.001 *$} \\
\hline & Above FL & $71.74 \pm 2.39$ & \\
\hline & Axial & $103.07 \pm 6.49$ & \\
\hline & Axio-occlousal & $127.76 \pm 3.99$ & \\
\hline & Occlusal & $164.87 \pm 4.11$ & \\
\hline
\end{tabular}

\section{DISCUSSION}

A metal die was milled to resemble the dimensions of reduced mandibular first molar with rounded shoulder finish line ${ }^{(5)}$. Resin dies were used to simulate tooth structure as they have similar modulus of elasticity to that of dentin $(12.9 \mathrm{GPa})^{(4)}$

The conventional lost wax technique includes multiple manual steps resulting in greater chance for errors ${ }^{(2,18)}$. While, CAD wax pattern technique aims to reduce inaccuracies by reducing production steps. The spacer thickness of CAD technology cannot be reduced below $50 \mu \mathrm{m}$. However, other factors could affect the internal fit such as abutment preparation design, scanning device accuracy, software design, and milling machine accuracy ${ }^{(2,3)}$. A light laser scanner tends to make sharp edges more rounded ${ }^{(2)}$. Thickness of wax was verified by wax caliper followed by casting with $\mathrm{NiCr}^{(7)}$.

Internal gap is the vertical distance from the axial surface of the die to the internal surface of restorations and assessment has clinical relevance 
affecting the strength of a restoration cement system ${ }^{(1)}$. Occlusal fit may play a significant role on structural durability of restorations and the axial wall internal fit can influence the retention of fixed prostheses ${ }^{(3)}$. Lack of adequate fit is potentially detrimental to abutment which might lead to cement solubility ${ }^{(2)}$. An internal fit ranging between 50 to $100 \mu \mathrm{m}$ has been considered acceptable. Internal fit was measured by silicon replica as it is considered a reliable technique for in-vitro studies. It is a nondestructive technique that does not cause damage to the resin die or restoration ${ }^{(11,15)}$.

Null hypothesis was partially accepted as results revealed that there were insignificant differences in internal fit of conventional lost wax and CAD wax technique. However, the other part of null hypothesis was rejected as there was significant difference between different measuring sites of internal fit for both constructed techniques.

Insignificant difference in internal gap values between conventional wax $(103.89 \mu \mathrm{m} \pm 40.39)$ and CAD wax specimens $(104.10 \mu \mathrm{m} \pm 39.96)$ might be because inaccuracies were equally weighed in both groups. This was found to be in agreement with some studies ${ }^{(1,2,5)}$. The results of the study are in contradiction with Farjood et al., ${ }^{(3)}$ where CAD wax experienced greater discrepancy probably due to use four axis milling machine. However restoration quality is related to digitalization of scanner, software processing and milling process rather than the number of processing axes. Scanner might induce wider internal gaps due to use of antireflection spray ${ }^{\left({ }^{8}\right)}$.

Moreover, results showed that there was a significant difference between internal gap values measured at different positions. The highest internal gap was found at the occlusal surface followed by axio-occlusal area, while the lowest gap value was found at the finish line. Occlusal site might have highest inaccuracy due to anatomical morphology which may not allow the flow of the replica with accumulation of die spacer in slope of the prepared cusps in conventional technique (2). Also, high internal gap values at axio-occlusal site might be due to inability of milling machine to compensate for drill compensation phenomenon (2). Precision of internal fit depended on the size of the smallest usable tool of system ${ }^{(1)}$. While internal gap value was lowest at finish line as convergence in preparation allows replica to flow easily ${ }^{(7,8)}$. The results of current study are in agreement with some studies ${ }^{(1,8)}$ . The results of this study are in contradiction with Colpani et al., ${ }^{(6)}$ who found that metal alloy casting uniformly shrinks at all measured regions.

Limitations of the present study that it is an in vitro study, not all in vivo simulation conditions were applied like mechanical forces, $\mathrm{pH}$ changes, presence of saliva and bacteria. Silicon replica technique provides two dimensional measurements.

\section{CONCLUSIONS}

Within the limitation of this study the following conclusions could be drawn:

1. The CAD technique for wax pattern showed comparable internal fit to conventional wax technique.

2. Considering different measuring points of internal fit for both construction techniques, occlusal areas showed the highest significant discrepancy compared to the other areas.

3. Both construction techniques of casting were considered reliable in restoration production within a clinically acceptable range regarding internal fit.

\section{REFERENCES}

1. Park JY, Kim HY, Kim JH, Kim JH, Kim WC: Comparison of prosthetic models produced by traditional and additive manufacturing methods. J Adv Prosthodont 2015; 7:294-302

2. Fathi H, Al-Masoody A, El-Ghezawi N, Johnson A: The Accuracy of Fit of Crowns Made From Wax Patterns Produced Conventionally (Hand Formed) and Via CAD/CAM Technology. J Prosthodont Restor Dent 2016; 24:7-10 
3. Farjood E, Vojdani M, Torabi K, Khaledi AR: Marginal and internal fit of metal copings fabricated with rapid prototyping and conventional waxing. J Prosthet Dent 2017; 117:164-170.

4. Munoz S, Ramos VJ, Dickinson DP: Comparison of margin discrepancy of complete gold crowns fabricated using printed, milled, and conventional hand-waxed patterns. J Prosthet Dent 2017; 118:89-94.

5. Lalande D, Hodd JA, Brousseau JS, Ramos V, Dunham D, Rueggeberg F: Marginal discrepancy dimensions of single unit metal crowns fabricated by using CAD-CAM milled acrylate resin polymer blocks or a conventional waxing technique. J Prosthet Dent 2018; 119:948-953.

6. Colpani JT, Borba M, Bona ÁD: Evaluation of marginal and internal fit of ceramic crown copings. J Dent Mater 2013; 29:174-180.

7. Nesse H, Ulstein DMA, Vaage MM, Øilo M: Internal and marginal fit of cobalt-chromium fixed dental prostheses fabricated with 3 different techniques. J Prosthet Dent 2015; 114:686-692.

8. Park JK, Lee WS, Kim HY, Kim WC, Kim JH: Accuracy evaluation of metal copings fabricated by computer-aided milling and direct metal laser sintering systems. J Adv. Prosthodont 2015; 7(2):122-128.

9. Kim EH, Lee DH, Kwon SM, Kwon TY: A microcomputed tomography evaluation of the marginal fit of cobaltchromium alloy copings fabricated by new manufacturing techniques and alloy systems. J Prosthet Dent 2017; 117:393-399.

10. Ghodsi S, Pirmoazen S, Beyabanaki E, Alikhasi M: The Effect of Milling Metal Versus Milling Wax on Implant Framework Retention and Adaptation: Milling Metal Versus Milling Wax. J Prosthodont 2018; 28(2)320-326.
11. Son K, Lee S, Kang SH, Park J, Lee KB, Jeon M and YunJ BJ.: A Comparison Study of Marginal and Internal Fit Assessment Methods for Fixed Dental Prostheses. J Clin. Med 2019; 8:785-790.

12. Varol S, \& Kulak-Ozkan Y: In Vitro Comparison of Marginal and Internal Fit of Press-on-Metal Ceramic (PoM) Restorations with Zirconium-Supported and Conventional Metal Ceramic Fixed Partial Dentures Before and After Veneering. J Prosthodont 2015; 24:387-393.

13. Rosella D, Rosella G, Brauner E, Papi P, Piccoli L, Pompa G:A tooth preparation technique in fixed prosthodontics for students and neophyte dentists. 2015; 6(4): 104-109.

14. Faul F, Erdfelder E, Lang AG, Buchner A: G*Power 3: A flexible statistical power analysis program for the social, behavioral, and biomedical sciences. Behavior Research Methods.2007; 39, 175-191

15. Mai HN, Lee KE, Ha JH, Lee DH: Effects of image and education on the precision of the measurement method for evaluating prosthesis misfit. J. Prosthet. Dent. 2018;119:600-605.

16. Hasanza M, Shirani M., Afrashtehfar K, Naseri P, Alikhasi M: In Vivo and In Vitro Comparison of Internal and Marginal Fit of Digital and Conventional Impressions for FullCoverage Fixed Restorations: A Systematic Review and Meta-analysis. J Evid-Based Dent Pr 2019; 19(3):236-254.

17. Lövgren N, Roxner R, Klemendz S, Larsson C: Effect of production method on surface roughness, marginal and internal fit, and retention of cobalt-chromium single crowns. J Prosthet Dent 2017; 118:95-101.

18. Prabhu R, Prabhu G, Baskaran E, Arumugam EM: Clinical acceptability of metal ceramic fixed partial dental prosthesis fabricated with direct metal laser sintering technique-5 year follow-up. J Indian Prosthodont Soc 2016; 16:193-197. 